CALT-68-2348

\title{
Remarks On A-branes, Mirror Symmetry, And The Fukaya Category
}

\author{
Anton Kapustin* Dmitri Orlov ${ }^{\dagger}$
}

\begin{abstract}
We discuss D-branes of the topological A-model (A-branes), which are believed to be closely related to the Fukaya category. We give string theory arguments which show that A-branes are not necessarily Lagrangian submanifolds in the Calabi-Yau: more general coisotropic branes are also allowed, if the line bundle on the brane is not flat. We show that a coisotropic A-brane has a natural structure of a foliated manifold with a transverse holomorphic structure. We argue that the Fukaya category must be enlarged with such objects for the Homological Mirror Symmetry conjecture to be true.
\end{abstract}

${ }^{*}$ California Institute of Technology, Pasadena, CA 91125, E-mail: kapustin@theory.caltech.edu

${ }^{\dagger}$ Algebra Section, Steklov Mathematical Institute, Russian Academy of Sciences, 8 Gubkin str., GSP-1, Moscow 117966, Russia, E-mail: orlov@mi.ras.ru 


\section{Introduction}

Let $X$ be a weak Calabi-Yau manifold, i.e. a complex manifold with $c_{1}(X)=0$ which admits a Kähler metric. Given a Ricci-flat Kähler metric $G$ on $X$, and a B-field (a class in $\left.H^{2}(X, \mathbb{R})\right)$, one can canonically construct an $N=2$ supersymmetric sigma-model with "target" $X$. On physical grounds, the quantized version of this model has $N=2$ superconformal symmetry and describes propagation of closed strings on $X$. In this note we set $B=0$ for simplicity. According to Calabi's conjecture proved by Yau, we can parametrize $G$ by the cohomology class of its Kähler form $\omega$. A weak Calabi-Yau manifold equipped with a Kähler form $\omega$ will be called a physicist's Calabi-Yau.

It sometimes happens that two different physicist's Calabi-Yau manifolds $(X, \omega)$ and $\left(X^{\prime}, \omega^{\prime}\right)$ give rise to a pair of $N=2$ superconformal field theories (SCFTs) related by a mirror morphism [1, 2]. A mirror morphism of $N=2$ SCFTs is an isomorphism of the underlying $N=1$ SCFTs which acts on the $N=2$ super-Virasoro algebra as a mirror involution [3, 4. In this case one says that $(X, \omega)$ and $\left(X^{\prime}, \omega^{\prime}\right)$ are mirror to each other. (For a concise explanation of the notions involved and further references see [5]. An algebraically-minded reader may find it useful to consult Ref. [6] for a careful definition of $N=2$ SCFTs and their morphisms.)

A long-standing problem is to understand the mirror relation from a mathematical viewpoint, i.e. without a recourse to the ill-defined procedure of quantizing a sigma-model. A fascinating conjecture has been put forward by M. Kontsevich [7]. He observed that to any physicist's Calabi-Yau $(X, \omega)$ one can associate two triangulated categories: the well-known bounded derived category of coherent sheaves $D^{b}(X)$ and the still mysterious Fukaya category $D \mathcal{F}(X)$. Objects of the category $D^{b}(X)$ are bounded complexes of coherent sheaves. Objects of the Fukaya category are (roughly speaking) vector bundles on Lagrangian submanifolds of $X$ equipped with unitary flat connections. The Homological Mirror Symmetry Conjecture (HMSC) asserts [7] that if two algebraic physicist's Calabi-Yau manifolds $(X, \omega)$ and $\left(X^{\prime}, \omega^{\prime}\right)$ are mirror to each other, then $D^{b}(X)$ is equivalent to $D \mathcal{F}\left(X^{\prime}\right)$, and $D \mathcal{F}(X)$ is equivalent to $D^{b}\left(X^{\prime}\right)$. So far this conjecture has been proved only for elliptic curves [B].

From a physical viewpoint, complexes of coherent sheaves are D-branes of the topological B-model (B-branes). We remind that the B-model of a physicist's Calabi-Yau $(X, \omega)$ is a topological "twist" of the corresponding $N=2$ SCFT [9]. The twisted theory is a two-dimensional topological field theory whose correlators do not depend on $\omega$. Morphisms between the objects of $D^{b}(X)$ are identified with the states of the topological string stretched between pairs of B-branes, and the compositions of morphisms are computed by the correlators of the B-model. This correspondence has been intensively discussed in the 
physics literature (see for example 110, 11, 12, 13] and references therein), and will be taken as a starting point here.

An $N=2$ SCFT has another twist, called the A-twist [9]. The corresponding topological field theory (the A-model) is insensitive to the complex structure of $X$, but depends nontrivially on the symplectic form $\omega$. D-branes of the A-model are called A-branes. Mirror morphisms exchange A- and B-twists and A- and B-branes. Thus from a physical viewpoint the mirror of $D^{b}(X)$ is the category of A-branes on $X^{\prime}$.

It can be shown that any object of the Fukaya category gives rise to an A-brane. Moreover, the recipe for computing morphisms between such A-branes can be derived heuristically in the path integral formalism, and it reproduces the definition of morphisms in the Fukaya category [14]. Therefore the majority of researchers in the field assumed that the mirror relation between the categories of A- and B-branes is essentially a restatement of the HMSC in physical terms.]

In this note we will argue that this is not the case, because A-branes are not necessarily Lagrangian submanifolds in $X$. This was mentioned already in one of the first papers on the subject [15], but the general conditions for a D-brane to be an A-brane have not been determined there. In Section 3 we will show that a coisotropic submanifold of $X$ with a unitary line bundle on it is an A-brane if the curvature of the connection satisfies a certain algebraic condition. We remind that a submanifold $Y$ of a symplectic manifold $(X, \omega)$ is called coisotropic if the skew-complement of $\left.T Y \subset T X\right|_{Y}$ with respect to $\omega$ is contained in $T Y$. In the physical language, a coisotropic submanifold is a submanifold locally defined by first-class constraints. One can easily see that the dimension of a coisotropic submanifold is at least half the dimension of $X$, and that a middle-dimensional coisotropic submanifold is the same thing as a Lagrangian submanifold. Thus we show that the category of A-branes contains, besides Lagrangian A-branes, A-branes of larger dimension.

In Section 4 we explore the geometric interpretation of the algebraic condition on the curvature of the line bundle. We will see that an A-brane is naturally a foliated manifold with a transverse holomorphic structure. The notion of transverse holomorphic structure is a generalization of the notion of complex structure to foliated manifolds. If the space of leaves of a foliated manifold $Y$ is a smooth manifold, a transverse holomorphic structure on $Y$ is simply a complex structure on the space of leaves. The general definition is given in Section 4. In addition to being transversely holomorphic, a coisotropic A-brane also carries a transverse holomorphic symplectic form.

In the case of a Lagrangian A-brane, the foliation has codimension zero, there are no

\footnotetext{
${ }^{1}$ In fact, the calculation of morphisms between Lagrangian A-branes in Ref. 14 preceded the formulation of the HMSC and served as an important motivation for it.
} 
transverse directions, and the transverse holomorphic structure is not visible. In general, the foliation is determined by the restriction of $\omega$ to $Y$, while the transverse holomorphic structure comes from the curvature of the line bundle on the brane.

Interestingly, to prove that an A-brane has a natural transverse holomorphic structure, one needs to use some facts from bihamiltonian geometry. The subject matter of bihamiltonian geometry is manifolds equipped with two compatible (in a sense explained below) Poisson structures. In our case, the underlying manifold is foliated, and one is dealing with transverse Poisson structures. (If the space of leaves is a manifold, specifying a transverse Poisson structure is the same as specifying an ordinary Poisson structure on the space of leaves.) One of the transverse Poisson structures arises from the symplectic form $\omega$ in the ambient space $X$, and the other one from the curvature of the line bundle on $Y$.

Our understanding of the category of A-branes is far from complete. Nevertheless, it is clear that generally it includes objects other than Lagrangian submanifolds with flat vector bundles. (There are certain special, but important, cases where there seem to be no nonLagrangian A-branes, like the case of an elliptic curve, or a simply-connected Calabi-Yau 3-fold.) Therefore the Fukaya category must be enlarged with coisotropic A-branes for the HMSC to be true. (This is somewhat reminiscent of the remark made in [7] that Lagrangian foliations may need to be included in the Fukaya category.) This is discussed in more detail in Section 5.

Since our arguments are ultimately based on non-rigorous physical reasoning, a skeptic might not be convinced that the HMSC needs serious modification. To dispel such doubts, we discuss in Section 2 mirror symmetry for tori and show that under mild assumptions the usual Fukaya category cannot capture the subtle behavior of $D^{b}(X)$ under the variation of complex structure. Inclusion of coisotropic A-branes seems to resolve the problem.

\section{Why Lagrangian submanifolds are not enough}

In this section we give some examples which show that the Fukaya category must be enlarged with non-Lagrangian objects for the HMSC to be true. We will exhibit a mirror pair of tori such that mirror symmetry takes a holomorphic line bundle (a B-brane) on the first torus to a complex line bundle on the second torus. This means that the latter line bundle is an A-brane.

It is well known that the derived category of coherent sheaves behaves in a very nontrivial manner under a variation of complex structure, and at special loci in the moduli space of complex structures it can become "larger." This is easy to see on the level of the 
Grothendieck group of $D^{b}(X)$, which we denote by $K_{0}\left(D^{b}(X)\right)$. There is a map

$$
c h: K_{0}\left(D^{b}(X)\right) \otimes \mathbb{Q} \longrightarrow H^{*}(X, \mathbb{Q})
$$

called the Chern character. The image of this map is contained in the intersection of $H^{*}(X, \mathbb{Q})$ with $\oplus_{p} H^{p, p}(X)$ in the complex cohomology group $H^{*}(X, \mathbb{C})$ and, by the Hodge Conjecture, should coincide with this intersection.

Let us denote by $N S(X)$ the Neron-Severi group of $X$ which, by definition, is the image of a natural map from the Picard group $\operatorname{Pic}(X)$ to $H^{2}(X, \mathbb{Z})$. Then we have $N S(X) \otimes \mathbb{Q}=$ $\operatorname{Im}(c h) \cap H^{2}(X, \mathbb{Q})$, and therefore $\operatorname{Im}(c h)$ contains a subring generated by the NeronSeveri group.

One can see from examples that the image of the map ch can change under a variation of complex structure; in particular, the dimension of $\operatorname{Im}(\mathrm{ch})$ can jump if, for example, the dimension of the Neron-Severi group jumps.

The "jumping" phenomenon can be easily observed in the case of abelian varieties. Let $E_{\tau}$ be an elliptic curve with a Teichmüller parameter $\tau$. It has a structure of an algebraic group. Let $e$ be the identity point of this group. It can be checked that any endomorphism of $E_{\tau}$ that sends the point $e$ to itself is an endomorphism of the algebraic group. Such endomorphisms form a ring which contains $\mathbb{Z}$ as a subring and for a "generic" elliptic curve coincides with it. However the ring of $e$-preserving endomorphisms of $E_{\tau}$ can be bigger than $\mathbb{Z}$. In this case one says that the elliptic curve $E_{\tau}$ possesses a complex multiplication. It can be shown that $E_{\tau}$ has a complex multiplication iff $\tau$ is a root of a quadratic polynomial with integral coefficients. For example, the elliptic curve with $\tau=i$ is an example of a curve with a complex multiplication.

Let $E_{\tau}$ be an elliptic curve with a complex multiplication. Consider an $n$-dimensional abelian variety $A=E_{\tau}^{n}$ with $n \geq 2$. In this case the derived category $D^{b}(A)$ is in a certain sense much bigger than the derived category of a "generic" abelian variety. For a "generic" abelian variety the Neron-Severi group is $\mathbb{Z}$ and, moreover, $N S(A) \otimes \mathbb{Q}$ generates the whole $\operatorname{Im}(c h)$. Thus the dimension of $\operatorname{Im}(c h)$ is equal to $n+1$. For an abelian variety $E_{\tau}^{n}$, where $E_{\tau}$ is a "generic" elliptic curve, the dimension of the Neron-Severi group is $n(n+1) / 2$. If the elliptic curve posesses a complex multiplication, then $\operatorname{dim} N S(A)=n^{2}$ and, moreover, we have an equality

$$
\operatorname{Im}(\text { ch }) \otimes \mathbb{C}=\bigoplus_{p} H^{p, p}
$$

Thus in this case $\operatorname{dim}_{\mathbb{Q}} \operatorname{Im}($ ch $)=\left(\begin{array}{c}2 n \\ n\end{array}\right)$.

For example, for $n=2$, if $\tau$ is generic, the Neron-Severi group has dimension 3 and is generated by the divisors $\{p t\} \times E_{\tau}, E_{\tau} \times\{p t\}, \Delta$, where $\Delta$ is the diagonal of $E_{\tau} \times E_{\tau}$. 
In contrast, when $E_{\tau}$ posesses complex multiplication, $N S(A)$ has dimension 4, which coincides with the dimension of $H^{1,1}(A)$. It is generated by the divisors $\{p t\} \times E_{\tau}, E_{\tau} \times$ $\{p t\}, \Delta, \Gamma$, where $\Gamma \subset E_{\tau} \times E_{\tau}$ is the graph of an additional endomorphism of $E_{\tau}$.

Now let us look at the Fukaya category of a mirror torus. The mirror relation for abelian varieties is well-understood [16, 6] (see also [17]). In particular, it is known that for any abelian variety $A$ one can find a symplectic form $\omega$ such that for the pair $(A, \omega)$ there exists a mirror-symmetric abelian variety $B$ with a symplectic form $\omega_{B}$ ([16], Prop. 9.6.1). Let $D \mathcal{F}\left(B, \omega_{B}\right)$ be the Fukaya category of the symplectic manifold $\left(B, \omega_{B}\right)$. This category essentially depends only on the symplectic form $\omega_{B}$ and does not depend on the complex structure of the variety $B$. This is mirror to the obvious fact that the derived category of coherent sheaves does not depend on the symplectic form. By the HMSC the category $D \mathcal{F}\left(B, \omega_{B}\right)$ should be equivalent to the derived category $D^{b}(A)$.

Furthermore, the mirror correspondence induces an isomorphism of the cohomology vector spaces

$$
\beta: H^{*}(A, \mathbb{Q}) \stackrel{\sim}{\longrightarrow} H^{*}(B, \mathbb{Q}) .
$$

For abelian varieties the isomorphism $\beta$ is described in [16]. It is natural to assume that $\beta$ is compatible with the conjectured equivalence between the derived category $D^{b}(A)$ and the Fukaya category $D \mathcal{F}\left(B, \omega_{B}\right)$. This means that there should exist a map $\phi$ from the Grothendieck group $K_{0}\left(D \mathcal{F}\left(B, \omega_{B}\right)\right) \otimes \mathbb{Q}$ to the cohomology group $H^{*}(B, \mathbb{Q})$ which closes the commutative diagram

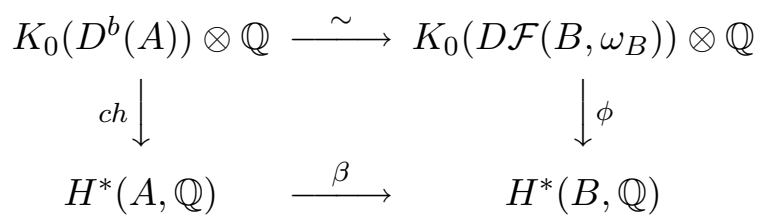

Under the map $\phi$ a flat vector bundle on a Lagrangian submanifold goes to the corresponding cycle in the middle-dimensional cohomology group $H^{n}(B, \mathbb{Q})$ with a multiplicity equal to the rank of the bundle.

Now note that classes of Lagrangian submanifolds in the middle-dimensional cohomology group belong to the kernel of a surjective map

$$
H^{n}(B, \mathbb{C}) \stackrel{\cdot\left[\omega_{B}\right]}{\longrightarrow} H^{n+2}(B, \mathbb{C}) .
$$

The dimension of the kernel is equal to $\left(\begin{array}{c}2 n \\ n\end{array}\right)-\left(\begin{array}{c}2 n \\ n+2\end{array}\right)$, which is less than the dimension of $\operatorname{Im}(c h)$. Therefore when $A=E_{\tau}^{n}$, where $E_{\tau}$ is an elliptic curve with a complex multiplication, Lagrangian submanifolds in $B$ with flat vector bundles can not generate the mirror of $D^{b}(A)$, in contradiction with the HMSC. 
To obtain some information on the mysterious mirror of $D^{b}(A)$, let us describe the mirror symmetry correspondence for $A=E_{\tau}^{n}$ more explicitly. In this case mirror symmetry is a T-duality. For simplicity we let $\tau=i$, so that $E_{\tau}$ is a "square torus." Consider a decomposition of the lattice $H^{1}(A, \mathbb{Z})=\Gamma \oplus \Sigma$ with bases $\Gamma=\left\langle x_{1}, \ldots, x_{n}\right\rangle$ and $\Sigma=$ $\left\langle y_{1}, \ldots, y_{n}\right\rangle$ such that the complex structure $I_{A}$ takes $x_{i}$ to $y_{i}$ and $y_{i}$ to $-x_{i}$. Let $\left\langle l_{1}, \ldots, l_{n}\right\rangle$ be the dual basis in the dual lattice $\Gamma^{*}$. A mirror manifold for the abelian variety $A$ can be constructed by T-dualizing the directions $x_{1}, \ldots, x_{n}$. This means that the mirror manifold $B$ is a torus $\left(\Gamma^{*} \oplus \Sigma\right) \otimes \mathbb{R} /\left(\Gamma^{*} \oplus \Sigma\right)$ equipped with a constant symplectic form

$$
\omega_{B}=\sum_{i=1}^{n} l_{i} \wedge y_{i} .
$$

(For simplicity we do not introduce a symplectic form on $A$ and a complex structure on B.)

In this case the map $\beta$ is defined in the following way. Let $T$ be a real $3 n$-dimensional torus $\Pi \otimes \mathbb{R} / \Pi$, where $\Pi=\Gamma \oplus \Sigma \oplus \Gamma^{*}$. The torus $T$ has natural projections $p$ and $q$ to the tori $A$ and $B$ :

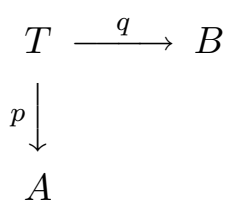

Let $P$ be a complex line bundle on $T$ defined by its first Chern class:

$$
c_{1}(P)=\sum_{i=1}^{n} x_{i} \cdot l_{i} .
$$

The Chern character $\operatorname{ch}(P) \in H^{*}(T, \mathbb{Q})$ is equal to $\exp \left(c_{1}(P)\right)$. According to [16], the map $\beta$ from $H^{*}(A, \mathbb{Q})$ to $H^{*}(B, \mathbb{Q})$ is given by the formula

$$
\beta(a):=q_{*}\left(\operatorname{ch}(P) \cdot p^{*}(a)\right) .
$$

(To define the map $q_{*}$ we chose fundamental classes of $T$ and $B$ and used the Poincare duality between cohomology and homology groups). Using this formula, one can explicitly calculate the subspace $\beta(\operatorname{Im}(c h))$.

To demonstrate the existence of objects in the mirror of $D^{b}(A)$ which are not Lagrangian submanifolds, we let $n=2$ for simplicity and consider a holomorphic line bundle $L$ on $A$ whose first Chern class is equal to

$$
c_{1}=x_{1} \cdot x_{2}+y_{1} \cdot y_{2}
$$

Such a holomorphic line bundle exists because $c_{1} \in H^{1,1}(A)$. The moduli space of such holomorphic line bundles is a homogeneous space over $\operatorname{Pic}^{0}(A)$, the kernel of the natural 
map from $\operatorname{Pic}(A)$ to $N S(A)$. More explicitly, $L$ is of the form $\mathcal{O}(-D) \otimes N$, where $N \in \operatorname{Pic}^{0}(A), \quad D=\Gamma-\{p t\} \times E-E \times\{p t\}$, and $\Gamma$ is the graph of the automorphism of $E$ given by multiplication by $i$. A direct calculation shows that

$$
\beta(\operatorname{ch}(L))=\left(1 ; y_{1} \cdot y_{2}-l_{1} \cdot l_{2} ;-y_{1} \cdot y_{2} \cdot l_{1} \cdot l_{2}\right) \in H^{\text {even }}(B, \mathbb{Q}) .
$$

We see that $\beta(\operatorname{ch}(L))$ coincides with the Chern character $\operatorname{ch}(M)$ of a complex line bundle $M$ on $B$ with the first Chern class equal to

$$
c_{1}(M)=y_{1} \cdot y_{2}-l_{1} \cdot l_{2}
$$

Therefore it is natural to expect that the complex line bundle $M$ (with an unitary connection) is an object of the mirror of $D^{b}(A)$, and that the invertible coherent sheaf $L$ goes to the line bundle $M$ under the mirror symmetry correspondence described above. In physical terms, this shows that the mirror of a D4-brane of type B with a flux wrapped on a 4-torus can be a D4-brane of type A with a flux wrapped on the mirror torus.

One can check that in this case the subspace $\beta(\operatorname{Im}(c h))$ consists of the elements $(r ; c ; s) \in H^{\text {even }}(B, \mathbb{Q})$ such that

$$
c \cdot \omega_{B}=0, \quad s=\frac{1}{2} r \omega_{B}^{2} .
$$

Similarly, for any $n>2$ we can find elements of $\beta(\operatorname{Im}(c h))$ which do not belong to the middle cohomology group of $B$ and therefore correspond to non-Lagrangian objects of the mirror of $D^{b}(A)$.

One may ask how general this phenomenon is. It does not occur for odd-dimensional Calabi-Yau manifolds which are complete intersections in projective spaces. But it seems that for even-dimensional Calabi-Yaus (for example, for K3 surfaces) or for more general odd-dimensional Calabi-Yaus the situation is similar to that for abelian varieties, i.e. nonLagrangian A-branes appear at special points in the moduli space of symplectic structures.

\section{World-sheet approach to A-branes}

This section assumes some familiarity with supersymmetric sigma-models (on the classical level) and superconformal symmetries. Let $X$ be a Kähler manifold with metric $G$ and Kähler form $\omega$. The complex structure on $X$ is given by $I=G^{-1} \omega$. The supersymmetric sigma-model with target $X$ classically has $(2,2)$ superconformal symmetry. Quantum anomaly destroys this symmetry unless $c_{1}(X)=0$.

Let $j: Y \rightarrow X$ be a submanifold in $X$, and $E$ be a line bundle on $Y$ with a unitary connection. Our goal is to derive the necessary and sufficient conditions for a pair $(Y, E)$ 
to be a D-brane of type A. We will find that these conditions depend on $\omega$, but are not sensitive to the complex structure on $X$, as expected on general grounds.

Let $\mathcal{W}$ be an open string world-sheet, i.e. a Riemann surface with a boundary. The fields of the sigma-model consist of a smooth map $\Phi: \mathcal{W} \rightarrow X$, and sections $\psi, \bar{\psi}$ of $\Phi^{*}(T X) \otimes \Pi S^{ \pm}$. Here $S^{ \pm}$are semi-spinor line bundles on $\mathcal{W}$, and $\Pi$ is the parity-reversal functor. In the physical language, $\Phi$ is a bosonic field, while $\psi$ and $\bar{\psi}$ are fermionic fields. The precise form of the action is unimportant for our purposes; what is important is that the action has $(2,2)$ superconformal symmetry. In particular, the supercurrents $Q^{ \pm}, \bar{Q}^{ \pm}$ are given by

$$
\begin{aligned}
Q^{ \pm} & =\frac{i}{4 \sqrt{2}} G(\psi, \partial \Phi) \pm \frac{1}{4 \sqrt{2}} \omega(\psi, \partial \Phi), \\
Q^{ \pm} & =\frac{i}{4 \sqrt{2}} G(\bar{\psi}, \bar{\partial} \Phi) \pm \frac{1}{4 \sqrt{2}} \omega(\bar{\psi}, \bar{\partial} \Phi),
\end{aligned}
$$

and the $U(1)$ R-currents are given by

$$
\begin{aligned}
J & =-\frac{i}{2} \omega(\psi, \psi), \\
\bar{J} & =-\frac{i}{2} \omega(\bar{\psi}, \bar{\psi}) .
\end{aligned}
$$

Supercurrents and R-currents are sections of powers of the semi-spinor bundles.

Consider open strings ending on $Y$, i.e. maps $\Phi$ such that some or all of the components of $\partial \mathcal{W}$ are mapped to $Y$. For example, we may consider the situation where $\mathcal{W}$ is an upper half-plane, and $\partial \mathcal{W}$ is the real axis. Then the map $\Phi$ and the sections $\psi, \bar{\psi}$ must satisfy on the boundary $z=\bar{z}$ the following conditions:

$$
\begin{aligned}
\partial \Phi & =R(\bar{\partial} \Phi) \\
\psi & =R(\bar{\psi}) .
\end{aligned}
$$

Here $R$ is an endomorphism of the restriction of $T X$ to $Y$. Furthermore, $R$ can be expressed in terms of $G$ and the curvature of the line bundle $E$. To write it down, we will use the metric $G$ to decompose $\left.T X\right|_{Y}$ as $N Y \oplus T Y . \quad R$ preserves this decomposition and has the form

$$
R=\left(-i d_{N Y}\right) \oplus(g-F)^{-1}(g+F) .
$$

Here $g$ is the restriction of $G$ to $Y$, and $F$ is the curvature 2-form of the line bundle $E$. (We use the physical convention in which $F$ is real.)

The physical meaning of this formula is very simple. Recall that the boundary of the string world-sheet $\mathcal{W}$ is the trajectory of a string end-point, and that the string end-point 
is charged with respect to the gauge field on the brane [18]. Thus for non-zero $F$ there is a Lorenz force acting on the end-point. Eqs. (2) and (4) say that the velocity of the end-point is tangent to $Y$, and that the Lorenz force acting on it is balanced by the string tension. Eq. (3) arises from the requirement of $N=1$ world-sheet supersymmetry.

It is easy to check that $R$ satisfies

$$
R^{t} G R=G,
$$

i.e. $R$ is an orthogonal transformation of $\left.T X\right|_{Y}$. This implies that on the boundary the left-moving and right-moving $N=1$ supercurrents are equal:

$$
Q^{+}+Q^{-}=\bar{Q}^{+}+\bar{Q}^{-} \text {. }
$$

Thus such a boundary condition automatically preserves $N=1$ superconformal symmetry and therefore corresponds to a D-brane [18].

Boundary conditions for a topologically twisted sigma-model must in addition preserve $N=2$ superconformal symmetry [14]. This can be achieved in two inequivalent ways: either we must have

$$
Q^{ \pm}=\bar{Q}^{ \pm}, \quad J=\bar{J}
$$

or

$$
Q^{ \pm}=\bar{Q}^{\mp}, \quad J=-\bar{J},
$$

on the boundary. In the first case we say that we have a B-type boundary condition, while in the second case we have an A-type boundary condition. One can show that a B-type boundary condition corresponds to a B-brane, while an A-type boundary condition corresponds to an A-brane [14].

It is easy to see that $R$ corresponds to a B-type boundary condition if and only if $R^{t} \omega R=\omega$. Since $R$ is orthogonal, this is equivalent to saying that $R$ commutes with the complex structure $I=G^{-1} \omega$. The latter condition obviously implies that $Y$ is a complex submanifold in $X$, and, less obviously, that $F$ is of type $(1,1)$. Thus a B-brane is a complex submanifold in $X$ with a holomorphic line bundle. This is the standard result [14, 15].

On the other hand, $R$ corresponds to an A-type boundary condition if and only if

$$
R^{t} \omega R=-\omega
$$

To analyze this equation, let us choose a basis in $\left.T X\right|_{Y}$ in which the first $\operatorname{dim}_{\mathbb{R}} X-$ $\operatorname{dim}_{\mathbb{R}} Y$ vectors span $N Y$ and the remaining $\operatorname{dim}_{\mathbb{R}} Y$ vectors span $T Y$. Let $\omega^{-1}$ have the following form in this basis

$$
\omega^{-1}=\left(\begin{array}{cc}
A & B \\
-B^{t} & C
\end{array}\right)
$$


where $A=-A^{t}, C=-C^{t}$. Then the condition Eq. (5) is equivalent to the following conditions on $A, B, C$ :

$$
\begin{aligned}
A & =0, \\
B F & =0, \\
g C g & =F C F .
\end{aligned}
$$

The first condition means that $Y$ is a coisotropic submanifold of $X$. This implies that $\left.\omega\right|_{Y}$ has a constant rank, and the dimension of the bundle $\mathcal{L} Y=\operatorname{ker}\left(\left.\omega\right|_{Y}\right)$ is equal to the codimension of $Y$.

The second condition is equivalent to the statement that if we regard the 2 -form $F$ as a bundle morphism $T Y \rightarrow T Y^{*}$, then its restriction to $\mathcal{L} Y$ vanishes. In other words, if we denote by $\mathcal{F} Y$ the quotient bundle $T Y / \mathcal{L} Y$, then $F$ descends to a section of $\Lambda^{2} \mathcal{F} Y$. We will denote this section $f$. The form $\omega$ gives rise to another section of $\Lambda^{2} \mathcal{F} Y$, which we will call $\sigma$. Obviously, $\sigma$ is non-degenerate and makes $\mathcal{F} Y$ into a symplectic bundle (i.e. a vector bundle with a smoothly varying symplectic structure on the fibers).

Now let us analyze the third condition. The metric $g$ provides a canonical splitting $T Y=\mathcal{L} Y \oplus \mathcal{F} Y$, and it is easy to see that $C$ is simply $0 \oplus \sigma^{-1}$. The Kähler property of the metric then implies

$$
g C g=0 \oplus(-\sigma)
$$

and therefore the third condition is equivalent to

$$
f \sigma^{-1} f=-\sigma
$$

In other words, if we denote the endomorphism $\sigma^{-1} f: \mathcal{F} Y \rightarrow \mathcal{F} Y$ by $J$, then $J^{2}=-1$. Thus $\mathcal{F} Y$ has a natural complex structure.2

An obvious consequence of the first condition is that $\operatorname{dim}_{\mathbb{R}} Y-\frac{1}{2} \operatorname{dim}_{\mathbb{R}} X$ is a nonnegative integer. The other two conditions imply that this integer is even. Indeed, the complex structure $J$ leads to the Dolbeault decomposition of $\Lambda^{2} \mathcal{F} Y$, and it is easy to see that both $\sigma$ and $f$ are forms of type $(0,2)+(2,0)$. Since both forms are non-degenerate, it follows that the complex dimension of $\mathcal{F} Y$ must be even. This in turn implies that $\operatorname{dim}_{\mathbb{R}} Y-\frac{1}{2} \operatorname{dim}_{\mathbb{R}} X$ is even.

For example, when $X$ is a 4-dimensional manifold ( $T^{4}$ or a K3 surface), an A-brane can be either 2-dimensional or 4-dimensional. When $X$ is 6-dimensional, an A-brane can be either 3-dimensional or 5-dimensional. Note that a Calabi-Yau 3-fold which is a complete

\footnotetext{
${ }^{2}$ Note that $\mathcal{F} Y$ is both a complex bundle and a symplectic bundle, but it is not a unitary bundle. The symplectic form $\sigma$ on the fibers has type $(0,2)+(2,0)$ in the complex structure $J$. Thus $\sigma J=f$ is a skew-symmetric pairing, rather than a Kähler metric.
} 
intersection in a projective space has $H_{5}(X, \mathbb{Z})=0$, and therefore any 5-dimensional Abrane must be homologically trivial. This seems to suggest that all A-branes are middledimensional in this case.

Let us consider two extreme cases. If $\operatorname{dim}_{\mathbb{R}} Y=\frac{1}{2} \operatorname{dim}_{\mathbb{R}} X$, then the first condition on $Y$ says that $Y$ is Lagrangian. Since $\mathcal{L} Y=T Y$ in this case, the second condition says that $F$ is zero, i.e. the line bundle $E$ is flat. The third condition is vacuous in this case. Thus a middle-dimensional A-brane is a Lagrangian submanifold with a flat unitary line bundle. This is the standard result [14, 15]

Another extreme case is $Y=X$. In this case $\mathcal{L} Y$ is the zero vector bundle, and the first two conditions are trivially satisfied. The bundle $\mathcal{F} Y$ coincides with $T X$, and thus the third condition says that $J=\omega^{-1} F$ is an almost complex structure on $X$ :

$$
\left(\omega^{-1} F\right)^{2}=-i d
$$

We will see in the next section that $J$ is integrable, and thus $X$ is a complex manifold. Note that $X$ has a complex structure $I$ to begin with, but the topological A-model is insensitive to it. Given an A-brane wrapping the whole $X$, one can construct a new complex structure $J$ out of $\omega$ and $F$. It is necessarily different from $I$, because $\omega$ has type $(1,1)$ with respect to $I$ and type $(2,0)+(0,2)$ with respect to $J$.

If $X$ is compact, the 2-form $F$ must have integer periods, and it is clear that the equation $\left(\omega^{-1} F\right)^{2}=-i d$ can be satisfied only for very special $\omega$. For example, if $X$ is a 4-torus and $\omega$ is generic, no line bundle on $X$ can be an A-brane. Presumably, this implies that generically all A-branes are Lagrangian submanifolds in $X$. But for some special $\omega$ there appear additional A-branes with $\operatorname{dim}_{\mathbb{R}} Y=4$.

Let us show that this "jumping" phenomenon is mirror to the one described in Section 2. Recall that in Section 2 we considered a complex torus $A$ of a very special kind ( $n$-th power of an elliptic curve with a complex multiplication, $n>1$ ). The Grothendieck group of $D^{b}(A)$ and its image in $H^{*}(A, \mathbb{Q})$ are unusually large. We also described a map $\beta$ from the rational cohomology of $A$ to the rational cohomology of its mirror $B$, and showed that in general the image of $\beta$ does not lie in the middle-dimensional cohomology of the mirror torus. For example, for $n=2$ the image of $\beta$ lies in the even cohomology, and it can happen that $\beta$ maps the Chern character of a coherent sheaf on $A$ to an element which looks like the Chern character of a complex vector bundle on $B$. We interpreted this as saying that the mirror of a coherent sheaf on $A$ can be a complex vector bundle on $B$. The Chern classes of such a vector bundle are not arbitrary, but must satisfy certain constraints; for $n=2$ these constraints are given by Eq. (1). When the rank of the bundle is 1 , we can compare these constraints with the algebraic constraint on the curvature Eq. (9). The 
condition (9) means that the rank of the matrix $F-i \omega$ is half the dimension of $X$. If we set $\operatorname{dim}_{\mathbb{R}} X=2 n$, then this implies that $n$ is even, and that the $n / 2+1$-st exterior power of $F-i \omega$ vanishes. For $n=2$ the latter condition is equivalent to

$$
F \wedge \omega=0, \quad F \wedge F=\omega \wedge \omega .
$$

On the level of cohomology, these conditions are the same as Eq. (11) in the special case $r=1$. A similar argument can be made for $n>2$.

\section{The geometry of A-branes}

In this section we discuss the geometry of a general coisotropic A-brane. We will see that it has some beautiful connections with bihamiltonian geometry and foliation theory.

A coisotropic submanifold $Y$ of a symplectic manifold $X$ has several equivalent definitions. The usual definition is that at any point $p \in Y$ the skew-orthogonal complement of $T Y_{p}$ is contained in $T Y_{p}$. Another popular definition is that $Y$ is locally defined by first-class constraints. In other words, locally $Y$ can be represented as the zero-level of a finite set of smooth functions on $X$ all of whose Poisson brackets vanish on $Y$.

For our purposes, yet another definition will be useful. A submanifold $Y$ is coisotropic if and only if the restriction of $\omega$ to $Y$ has a constant rank, and its kernel $\mathcal{L} Y \subset T Y$ is an integrable distribution. This means that the commutator of any two vector fields in $\mathcal{L} Y$ also belongs to $\mathcal{L} Y$.

By the Frobenius theorem, this induces a foliation of $Y$ such that the vector fields tangent to the leaves of the foliation are precisely the vector fields in $\mathcal{L} Y$. The dimension of the leaves is equal to the codimension of $Y$. We may call $\mathcal{L} Y$ the tangent bundle of the foliation. The quotient bundle $\mathcal{F} Y=T Y / \mathcal{L} Y$ is called the normal bundle of the foliation. (Elementary notions from foliation theory that we will need can be found in Chapter 1 of Ref. 19.)

If we interpret $Y$ as a first-class constraint surface in a phase space of a mechanical system, then the meaning of the above foliation can be understood as follows. First-class constraints lead to gauge symmetries. A leaf in $Y$ is precisely an orbit of a point under all gauge transformations. Formally, the reduced phase space $Y_{\text {red }}$ describing gauge-invariant degrees of freedom is the quotient of $Y$ by gauge transformations. In other words, $Y_{\text {red }}$ is the space of leaves of the foliation. However, this space in general does not have good properties, e.g. it need not be a manifold, or even a Hausdorff topological space. Generally, it is unclear how to define dynamics on $Y_{\text {red }}$.

Instead, Dirac instructed us to work with gauge-invariant observables on $Y$, i.e. with smooth functions on $Y$ which are locally constant along the leaves of the foliation. Such 
functions form a sheaf $\mathcal{O}_{\mathcal{F}}(Y)$, which we can regard as the structure sheaf of the foliated manifold $Y$. It plays the role of the (generally non-existent) sheaf of smooth functions on the space $Y_{\text {red }}$. Similarly, the sheaf of sections of $\mathcal{F} Y$ locally constant along the leaves of the foliation replaces the tangent sheaf of $Y_{\text {red }}$. We will denote this sheaf $\mathcal{T}_{\mathcal{F}}(Y)$.

An A-brane is a coisotropic submanifold $Y$ with an additional structure: a unitary line bundle $E$ on $Y$ whose curvature $F$ satisfies certain constraints. As explained in the previous section, this additional structure makes $\mathcal{F} Y$ into a complex vector bundle with complex structure $J$. It is easy to see that both $F$ and $\omega$ are constant along the leaves, i.e.

$$
\mathcal{L}_{u} F=\mathcal{L}_{u} \omega=0, \quad \forall u \in \Gamma(\mathcal{L} Y)
$$

Thus $J=\sigma^{-1} f$ is also constant along the leaves. This means that $J$ defines a transverse almost complex (TAC) structure on $Y$. TAC structure is an analogue of almost complex structure for foliated manifolds. In the case when $Y_{\text {red }}$ is a manifold, giving a TAC structure on $Y$ is the same as giving an almost complex structure on $Y_{\text {red }}$.

The "foliated" analogue of a complex manifold is a manifold with a transverse holomorphic structure (see e.g. 20] for a definition and discussion). If $Y_{\text {red }}$ is a manifold, a transverse holomorphic structure on $Y$ is simply a complex structure on $Y_{\text {red }}$. In general, the definition goes as follows. A codimension $2 q$ foliation on $Y$ is specified locally by a submersion $f: U \rightarrow \mathbb{R}^{2 q} \simeq \mathbb{C}^{q}$, where $U$ is a coordinate chart. $]^{3}$ On the overlap of two charts $U$ and $V$ the two respective submersions $f$ and $g$ are related by a transition diffeomorphism $\tau: f(U \cap V) \rightarrow g(U \cap V)$. A transverse holomorphic structure on $Y$ is specified by a collection of charts covering $Y$ such that all transition diffeomorphisms are bi-holomorphic.

The "foliated" analogue of the sheaf of holomorphic functions is the sheaf of functions which are locally constant along the leaves and holomorphic in the transverse directions. A remarkable feature of this sheaf is that for a compact $Y$ all its cohomologies are finitedimensional [21, 20]. Similarly, one can define transversely holomorphic bundles on $Y$, and again for compact $Y$ their sheaf cohomologies are finite-dimensional [20]. In general, properties of compact transversely holomorphic manifolds are very similar to those of compact complex manifolds.

It is easy to see that every transverse holomorphic structure gives rise to a TAC structure. A TAC structure which arises in this way is called integrable. The integrability condition for a TAC structure is the vanishing of the corresponding Nijenhuis torsion defined as follows. Let $u$ and $v$ be local sections of $\mathcal{T}_{\mathcal{F}}(Y)$. It is easy to see that the Lie bracket on $T Y$ descends to a Lie bracket on $\mathcal{T}_{\mathcal{F}}(Y)$, therefore the commutator $[u, v]$ is well defined. The

\footnotetext{
${ }^{3} \mathrm{~A}$ submersion is a smooth map whose derivative is surjective.
} 
Nijenhuis torsion $T(J)$ is a section of $\mathcal{F} Y \otimes \Lambda^{2} \mathcal{F} Y^{*}$ whose value on $u, v$ is defined to be

$$
T(J)=[J u, J v]-J[J u, v]-J[u, J v]+J^{2}[u, v] .
$$

In the case of a trivial foliation, this reduces to the standard definition of the Nijenhuis torsion of an almost complex structure.

Obviously, an integrable TAC structure has a vanishing Nijenhuis torsion, because in suitable coordinates $J$ is constant. Conversely, by analogy with the classical case, one expects that any TAC structure with a vanishing Nijenhuis torsion is integrable. Indeed, as noted in Ref. [21], this is a special case of a theorem proved by Nirenberg [22]. Thus there is a one-to-one correspondence between transverse holomorphic structures on a foliated manifold $Y$ and TAC structures on $Y$ with a vanishing Nijenhuis torsion.

A remarkable and non-obvious fact is that the TAC structure $J$ on an A-brane $Y$ is automatically integrable. Let us give a proof of this fact for the extreme case when $Y=X$ and the foliation is trivial (i.e. each leaf is a point). It is easy to extend the proof to general coisotropic A-branes.

First note that both $\omega$ and $F$ are symplectic structures on $X$. Furthermore, since $\omega^{-1} F$ has eigenvalues $\pm i, \quad \omega_{t}=\omega+t F$ is non-degenerate for any real $t$, and therefore is a symplectic structure as well. Hence its inverse is a Poisson structure for any real $t$. Now note that by virtue of $\left(\omega^{-1} F\right)^{2}=-i d$ the inverse has a very simple form

$$
\omega_{t}^{-1}=\left(1+t^{2}\right)^{-1}\left(\omega^{-1}+t F^{-1}\right) .
$$

Thus any linear combination of $\omega^{-1}$ and $F^{-1}$ is a Poisson structure on $X$. In the language of bihamiltonian geometry [23, 24], $\omega^{-1}$ and $F^{-1}$ are compatible Poisson structures on $X$. Now we can use the fundamental theorem of bihamiltonian geometry [23, 24] which says that if two Poisson structures $a$ and $b$ are compatible, and $a$ is non-degenerate, then the endomorphism $a^{-1} b: T X \rightarrow T X$ has a vanishing Nijenhuis torsion. This theorem implies that the Nijenhuis torsion of $J$ vanishes, and therefore $J$ is integrable.

For a general coisotropic A-brane one can use the same argument, but all objects are replaced by their foliated analogues: $T X$ is replaced by $\mathcal{F} Y$, functions on $X$ are replaced by functions locally constant along the leaves, Poisson structures are replaced by transverse Poisson structures, etc. One can check that the fundamental theorem of bihamiltonian geometry remains valid in the foliated case. In fact, the version of this theorem proved in [23] (Theorem 3.12) is valid in a very general setting, where the exterior differential complex of a smooth manifold is replaced by an arbitrary complex over a Lie algebra. The statement we need is a special case of this theorem.

We have shown that if there exists an A-brane with $Y=X$, then $J=\omega^{-1} F$ is a complex structure on $X$. Furthermore, one can easily see that $F+i \omega$ is a closed 2 -form on $X$ 
of type $(2,0)$ and maximal rank, i.e. a holomorphic symplectic form. Thus in the complex structure $J$ the manifold $X$ is a compact holomorphic symplectic manifold. If in addition $X$ admits a Kähler metric compatible with $J$, then $X$ is necessarily hyperkähler [25]. In general, $X$ need not be hyperkähler for an A-brane with $Y=X$ to exist.

\section{A-branes and Homological Mirror Symmetry}

We have shown that an A-brane is a coisotropic submanifold in $X$, and that it is naturally a foliated manifold with a transverse holomorphic structure. Now let us see how this fits in with the Homological Mirror Symmetry Conjecture.

As explained in Section 1, the mirror of the derived category is the category of A-branes. We have seen that in general the set of A-branes includes non-Lagrangian coisotropic branes, and therefore the Fukaya category must be enlarged with such A-branes for the Homological Mirror Symmetry Conjecture to be true. Of course, in some special cases there may be no non-Lagrangian A-branes, and the generalization we are proposing is vacuous. For example, there are no non-Lagrangian A-branes on an elliptic curve for dimensional reasons. It also seems likely that there are no non-Lagrangian A-branes on odd-dimensional Calabi-Yaus which are complete intersections in projective spaces, because any non-Lagrangian A-brane would be homologically trivial. Nevertheless, we believe that a uniform formulation of the Homological Mirror Symmetry Conjecture for all weak Calabi-Yau manifolds would be very illuminating. Let us see how one far one can go in this direction.

One immediately sees the following major difficulty. A Lagrangian A-brane can carry a flat vector bundle of rank $r$ higher than one. From a physical viewpoint, such an Abrane should be thought of as $r$ coincident A-branes of rank one. The same reasoning suggests that there exist coisotropic A-branes with higher rank bundles. However, it is not clear to us what the constraints on the connection are in this case, and whether a transverse holomorphic structure arises again. Thus we do not really understand all the objects in the enlarged Fukaya category.

We will ignore this difficulty and try instead to say something about morphisms between the objects we already know. Unfortunately, understanding morphisms between different A-branes is not much easier than understanding A-branes with higher rank bundles: the former question is just an "infinitesimal" form of the latter. Therefore we will focus on the endomorphisms of coisotropic A-branes.

To guess the right definition, let us look at the two extremes: Lagrangian A-branes and Abranes wrapping the whole $X$ (i.e. $Y=X$ ). The space of endomorphisms of a Lagrangian A-brane $Y$ is its Floer homology $H F_{*}(Y, \mathbb{C})$. This is hard to compute, but in many cases 
it coincides with the de Rham cohomology $H^{*}(Y, \mathbb{C})$. From a physical viewpoint, the de Rham cohomology is a classical approximation to the Floer homology; the two coincide when there are no world-sheet instanton contributions to the path integral computing the Floer differential [14].

Now suppose we have an A-brane $Y=X$. This means that there exists a unitary line bundle on $X$ with a connection 1-form $A$ whose curvature $F=d A$ satisfies

$$
\left(\omega^{-1} F\right)^{2}=-i d
$$

As explained in the previous section, this implies that $J=\omega^{-1} F$ is a complex structure on $X$. On general grounds, endomorphisms of an A-brane must have the structure of a graded vector space (in physical terms, the grading is given by the ghost charge). A natural guess is the Dolbeault cohomology $H^{0, *}(X)$ with respect to $J$.

As a simple check, note that degree one elements in the space of endomorphisms must parametrize infinitesimal deformations of the A-brane. In the present case, a deformation is a real 1-form $a$ such that the curvature of the connection 1-form $A+a$ satisfies Eq. (10) up to terms quadratic in $a$. This is equivalent to the condition

$$
(d a) J+J^{t}(d a)=0,
$$

i.e. $d a$ must be a form of type $(1,1)$. If we denote by $a^{\prime \prime}$ the $(0,1)$ part of $a$, then the latter condition is equivalent to $\bar{\partial} a^{\prime \prime}=0$. Thus $a^{\prime \prime}$ represents a class in $H^{0,1}(X)$. Since $a$ is real, the $(1,0)$ part of $a$ is determined by $a^{\prime \prime}$ (is complex conjugate to it). Thus there is a natural map from the space of deformations of an A-brane to $H^{0,1}(X)$.

We want to show that this map becomes one-to-one, if we quotient the space of deformations by deformations which are isomorphisms in the category of A-branes. Obviously, the usual infinitesimal gauge transformations $a=d f$, where $f$ is a real function on $X$, induce isomorphisms. However, this is not all. In the case of Lagrangian A-branes it is known that a flow along a Hamiltonian vector field on $X$ induces an isomorphism in the Fukaya category, and it is natural to assume that the same is true for more general coisotropic A-branes. If $h$ is a smooth real function on $X$, and $V_{h}=\omega^{-1} d h$ is the corresponding Hamiltonian vector field, then the induced deformation of the connection 1-form $A$ on $X$ is

$$
a=\mathcal{L}_{V_{h}} A=i_{V_{h}} F+d\left(i_{V_{h}} A\right)
$$

where $\mathcal{L}_{V}$ is the Lie derivative along $V$. Thus the most general deformation $a$ which is an isomorphism in the category of A-branes has the form

$$
a=i_{V_{h}} F+d f
$$


where $h$ and $f$ are arbitrary smooth real functions on $X$. Taking into account the relation $J=\omega^{-1} F$, this can be rewritten as

$$
a=-2 J^{t} d h+d f=\partial(f+2 i h)+\bar{\partial}(f-2 i h) .
$$

Let us denote by $\mathbf{E x t}^{1}$ the space of deformations of the A-brane modulo isomorphisms. It is easy to check that the map from the space of deformations to $H^{0,1}(X)$ descends to a well-defined map from $\mathbf{E x t}^{1}$ to $H^{0,1}(X)$, and that the latter map is an isomorphism of real vector spaces, as claimed.

With these two examples in mind, it is not hard to guess the right graded vector space for a general coisotropic A-brane. If $Y$ is a foliated manifold with a transverse holomorphic structure, recall that we denoted by $\mathcal{O}_{\mathcal{F}}(Y)$ the sheaf of complex functions on $Y$ which are locally constant along the leaves of the foliation and holomorphic in the transverse directions. We propose that the space of endomorphisms of a coisotropic A-brane $Y$ is the cohomology of the sheaf $\mathcal{O}_{\mathcal{F}}(Y)$.

It is trivial to see that our proposal is consistent with the two extreme cases considered above. For a Lagrangian A-brane, $\mathcal{O}_{\mathcal{F}}(Y)$ is simply the sheaf of locally constant complex functions on $Y$, and its cohomology coincides with the de Rham cohomology of $Y$. For $Y=X \quad \mathcal{O}_{\mathcal{F}}(Y)$ is the sheaf of holomorphic functions on $X$ (with respect to the complex structure $J$ ), and we again get agreement.

It would be very interesting to understand how morphisms between different coisotropic A-branes should be defined. At first sight, no suitable complex whose cohomology one could compute presents itself. Perhaps this is simply a lack of imagination on our part.

In general, it appears that a geometric definition of the category of A-branes is very cumbersome. Finding such a definition is akin to trying to define the category of holomorphic vector bundles on a complex manifold using the zeros of their holomorphic sections. A more promising approach is to look for an algebraic definition of A-branes, for example as modules over some non-commutative algebra associated to a symplectic manifold $X$. It seems likely that this non-commutative algebra is related to the deformation quantization of $X$. Similar ideas have been discussed in [26, 27].

\section{Acknowledgments}

We are grateful to Dan Freed for useful suggestions and to Ezra Getzler for pointing out the relevance of bihamiltonian geometry. Some preliminary results have been presented by A.K. at the Duality Workshop, ITP, Santa Barbara, June 18 - July 13, 2001. A.K. would like to thank Ron Donagi, Dan Freed, Ezra Getzler, Tony Pantev, and other participants 
for stimulating discussions, and the organizers for making this workshop possible. A.K. was supported in part by DOE grants DE-FG02-90ER40542 and DE-FG03-92-ER40701. D.O. was supported in part by RFFI grant 99-01-01144 and a grant for support of leading scientific groups $\mathrm{N}$ 00-15-96085. The research described in this publication was made possible in part by Award No RM1-2089 of the U.S. Civilian Research and Development Foundation for the Independent States of the Former Soviet Union (CRDF).

\section{References}

[1] B. R. Greene and M. R. Plesser, Duality in Calabi-Yau moduli space, Nucl. Phys. B338, 15 (1990).

[2] P. Candelas, X. C. de la Ossa, P. S. Green, and L. Parkes, A pair of Calabi-Yau manifolds as an exactly soluble superconformal theory, Nucl. Phys. B359, 21 (1991).

[3] L. Dixon, Lectures at 1987 ICTP summer workshop in High Energy Physics and Cosmology.

[4] W. Lerche, C. Vafa, and N. P. Warner, Chiral rings in $N=2$ superconformal theories, Nucl. Phys. B324, 427 (1989).

[5] C. Voisin, Mirror Symmetry, Providence, R.I.: American Mathematical Society (1999).

[6] A. Kapustin and D. Orlov, Vertex algebras, mirror symmetry, and D-branes: the case of complex tori, hep-th/0010293.

[7] M. Kontsevich, Homological algebra of mirror symmetry, alg-geom/9411018.

[8] A. Polishchuk and E. Zaslow, Categorical mirror symmetry: the elliptic curve, Adv. Theor. Math. Phys. 2, 443 (1998) math.AG/9801119.

[9] E. Witten, Mirror manifolds and topological field theory, Essays on mirror manifolds, p. 120-158, Hong Kong: Internat. Press (1992) hep-th/9112056.

[10] M. R. Douglas, D-branes, categories and $N=1$ supersymmetry, hep-th/0011017.

[11] C. I. Lazaroiu, Unitarity, D-brane dynamics and D-brane categories, hep-th/0102183.

[12] P. S. Aspinwall and A. E. Lawrence, Derived categories and zero-brane stability, JHEP 0108, 004 (2001) hep-th/0104147.

[13] D. Diaconescu, Enhanced D-brane categories from string field theory, JHEP 0106, 016 (2001) [hep-th/0104200. 
[14] E. Witten, Chern-Simons gauge theory as a string theory, hep-th/9207094.

[15] H. Ooguri, Y. Oz, and Z. Yin, D-branes on Calabi-Yau spaces and their mirrors, Nucl. Phys. B 477, 407 (1996) hep-th/9606112.

[16] V. Golyshev, V. Lunts, and D. Orlov, Mirror symmetry for abelian varieties, J. Alg. Geom. 10, 433-496 (2001) math.AG/9912003.

[17] Yu. Manin, Mirror symmetry and quantization of abelian varieties, math.AG/0005143.

[18] J. Polchinski, String theory, vol. 1,2, Cambridge, UK: University Press (1998).

[19] P. Tondeur, Geometry of foliations, Basel: Birkhäuser (1997).

[20] X. Gómez-Mont, Transversal holomorphic structures, J. Diff. Geom. 15, 161 (1980).

[21] T. Duchamp and M. Kalka, Deformation theory for holomorphic foliations, J. Diff. Geom. 14, 317 (1979).

[22] L. Nirenberg, A complex Frobenius theorem, Seminar on analytic functions, IAS, Princeton, p. 172-189 (1957).

[23] I. Dorfman, Dirac structures and integrability of nonlinear evolution equations, Chichester: Wiley (1993).

[24] F. Magri, Eight lectures on integrable systems, Integrability of nonlinear systems (Pondicherry, 1996), p. 256-296, Berlin - Heidelberg: Springer (1997).

[25] A. Beauville, Variétés Kähleriennes dont la prèmiere classe de Chern est nulle, J. Diff. Geom. 18, 755 (1984).

[26] R. Nest and B. Tsygan, The Fukaya type categories for associative algebras, Deformation theory and symplectic geometry (Ascona, 1996), p. 285-300, Dordrecht: Kluwer (1997).

[27] Y. Soibelman, Quantum tori, mirror symmetry, and deformation theory, math.QA/0011162. 